the splanchnic area. The right side of the heart is not distended with blood. In fat embolism, on the other hand, the right auricle and ventricle are dilated. There is a general venous stasis. The accumulation of an excessive amount of blood in any one region or organ is apparently due largely to the effect of gravity. Thus, if the foot of the board to which the animal is fastened is elevated, the intracranial sinuses are found at necropsy to be greatly distended with blood. This can be demonstrated by attempting to remove the brain before opening the thorax and abdomen.

This difference in the distribution of the blood in the body is to be expected. In peptone shock, the low blood pressure is due to a loss of tonus in the vessels of the splanchnic region, the vascular area of the lungs being undiminished. In fat embolism, the passage of the blood through the lungs is mechanically interfered with, the vascular tonus in the splanchnic region and elsewhere remaining normal.

5. Edema of the lungs appears to be a fairly constant accompaniment of fat experimental embolism, but has not been encountered in any marked degree in peptone shock.

6. In previous papers, ${ }^{1}$ attention was called to the exaggerated reaction to nicotin frequently observed in dogs in peptone and anaphylactic shock. This marked rise in blood pressure was obtained only when the nicotin caused a temporary dyspnea. It was suggested in explanation of this phenomenon that the rise was due to the mechanical effect of respiratory suction on the reservoir of blood in the liver, and not to any direct action of the nicotin on the vasomotor apparatus itself. It was also suggested that this mechanical effect of dyspnea might be of value in the treatment of any condition of low blood pressure in human patients in whom there was a large reservoir of blood in the liver. Blood accumulated in this organ can be drawn into the right side of the heart by respiratory suction because the veins involved do not readily collapse. The walls of those in the liver are better supported than those in the neck, for instance. The distance between the right auricle and the point of entrance of the hepatic vein into the inferior vena cava is short. The vena cava here is wide, and its walls are supported and prevented from collapsing by the central tendon of the diaphragm through which it passes and to which it is attached.

Porter ${ }^{2}$ has observed that in fat embolism, the induction of deep and rapid respiration brought about by increasing the carbon dioxid in inspired air leads to a rise in blood pressure of from 15 to $30 \mathrm{~mm}$. of mercury. Although I have not used carbon dioxid, I have, in a number of experiments, observed such a rise in blood pressure when the rate and depth of respiration were increased from any cause.

Hence in both peptone poisoning and experimental fat embolism, dyspnea causes a rise in blood pressure. But there are certain important differences in the character of the rise in the two conditions. 1. In peptone shock, the rise is sharp, and the pressure tends to drop almost to its former low level as soon as the respiration becomes normal; in fat embolism, the rise is gradual and more sustained. 2. In fat embolism, the same rise in blood pressure is seen as a result of the rapid respiration, which can hardly be called dyspnea, that follows removal of the anesthetic and

1. Simonds, J. P.: A Study of Low Blood Pressure Not Associated with Trauma or Hemorrhage, Arch. Int. Med., December, 1916, p. 848; with Trauma or Hemorrhage, Arch. Int. Med., December, 1916,
Anaphylactic Shock in Dogs, Jour. Infect. Dis., 1916, 19, 746.

2. Porter, W. T.: Boston Med. and Surg. Jour., 1917, 176, 699. the partial recovery of the animal from its effects. 3. A rise is also readily induced by vigorous artificial respiration by means of a bellows, an operation which actually produces a positive intrathoracic pressure. Neither of these methods has produced a rise in pressure in any animal in a condition of peptone shock.

In view of these marked differences in the character of the rise in blood pressure that follows the induction of dyspnea in peptone shock and experimental fat embolism, respectively, it may be suggested that the mechanism in the two cases is quite different. The probable mechanism in peptone shock has been set forth in the papers referred to. ${ }^{1}$ There is evidence, which will be presented in detail later, that, in fat embolism, dyspnea and vigorous artificial respiration (with a bellows) act by aiding in the mechanical dislodgment of fat droplets from the capillaries of the lungs. This increases the vascular area available for the passage of blood from the right to the left side of the heart and thence into the general circulation. This may not always be an unmixed advantage, however, for it may lead to fat embolism of the brain, and death.

\section{SERUM THERAPY FOR TRICHINOSIS *}

\author{
BENJAMIN SCHWARTZ, B.A., M.A. \\ WASHINGTON, D. C.
}

In a recent paper, Salzer ${ }^{1}$ records some observations and experiments with reference to trichinosis. Among other things he reports successful results with serum therapy and states emphatically that the injection of immune serum has both a prophylactic and a curative value. Briefly stated, Salzer's claims with respect to "immune serum" are as follows:

1. Animals fed with infected meat later than twentyfour hours after the administration of serum from a convalescent animal prove to be immune.

2. Infected meat mixed with immune serum does not produce trichinosis in animals to which it is fed.

3. Immune serum injected into animals suffering with the disease produces a curative effect.

The first two statements are fairly clear and easily subject to experimental verification. The last statement is obscure. One might suppose a curative action would involve destruction of the parasite. The author, however, does not indicate that the parasite itself is destroyed, and rather gives the impression that the curative effect is to be judged by the disappearance of the principal symptoms of the disease, a criterion not yet shown to be applicable in cases of experimental trichinosis.

In order to procure data on the effects of supposed immune serum on the resistance of animals to trichinosis, I performed the following experiment:

Serum was obtained from a rabbit about four months after the disease had been experimentally produced. A postmortem examination of the animal after the drawing of the blood revealed a moderate infection with encysted trichinae. The blood drawn from the rabbit was placed in an ice-box, and on the following day two guinea-pigs were injected subcutaneously with 1 c.c. and 2 c.c. of the serum, respectively. Forty-eight hours after the injection, the animals were for-

* From the laboratory of the Zoological Division, Bureau of Animal Industry, United States Department of Agriculture.

1. Salzer, B. F.: A Study of an Epidemic of Fourteen Cases of Trichinosis with Cures by Serum Therapy, The Journal A. M. A., Aug. 19, 1916, pp. 579, 580. Salzer also presented a report of his experiments at a meeting of the New York Academy of Medicine, the minutes of which are published in the Medical Record, New York,
$1917, \mathbf{9 1}, 261$. 
cibly fed small quantities of trichinous meat. A salad consisting of lettuce and infected meat was also fed the following day. Fifteen days after the first feeding one of the animals was killed, and its diaphragm was compressed between two thick slides and examined microscopically. Numerous larvae were found in the fluid surrounding the muscle, showing conclusively that the serum had no effect on the normal migrations of the larvae. The second guinea-pig was killed twentynine days after the first feeding, and the muscles were found to be heavily infested.

Another experiment bearing on the prophylactic effect of immune serum was performed as follows:

Blood was drawn from a guinea-pig two months after the fist feeding with trichinous pork. After the drawing of the biood, the animal was examined and the musculature found to be heavily infested. The day following the drawing of the blood, 2 c.c. of the serum were injected subcutaneously into a sound guinea-pig. Two months later this guinea-pig was fed trichinous meat. The animal died twenty-one days after feeding, and on examination the muscles showed a heavy infestation with the parasites.

From the foregoing results it is evident that the administration of serum from a convalescent animal did not produce any prophylactic effects, but, on the contrary, animals after injection with serum readily became infested when fed trichinous meat, and the life cycle of the parasite did not deviate from its issual course.

In order to determine whether "immune serum" has any effect on the development of the parasites when administered after the feeding of trichinous meat, the following experiment was performed:

Two guinea-pigs were fed trichinous meat, and two days after feeding, injected subcutaneously with 1 c.c. and 2 c.c. of serum, respectively, from the rabbit mentioned in the first experiment. The serum was injected three days after the drawing of the blood from which it was obtained. It was kept in an ice-box until used. One of the animals died twenty days after the feeding, and its muscles were found to be heavily infested with trichinae. The second animal was killed thirty-one days after feeding, and yielded similar results.

If Salzer's contention with regard to the prophylactic value of immune serum is to be sustained, it would seem that animals convalescent from the disease should not be liable to a fresh infection.

I exposed rats suffering with trichinosis to a supplementary infection by feeding them a second time with trichinous meat about thirty-five to forty days after the first feeding. In order to make absolutely certain that there would be no confusion between the parasites of the first and second infection, the animals were killed within five days after the second feeding, and the intestinal contents were examined. In all cases it was found that the parasites of the second infection had increased in size, and in some cases had already copulated, and that the uteri of the females were full of eggs. The intestinal parasites from the first infection could readily be distinguished from the more active younger parasites by their sluggishness, and by the presence of larvae in the uterus of the females.

Salzer's contention that infected meat mixed with immune serum does not produce trichinosis in animals to which it is fed was tested a number of times, serum being used from animals at various stages of the disease. The experiments are described below:

EXPERIMENT 1.-A guinea-pig was bled fourteen days after an experimental infection with trichinosis. A postmortem examination revealed numerous parasites in the blood. The serum from the blood kept over night in an ice-box was thoroughly mixed with trichinous meat and lettuce in the form of a salad and fed to two guinea-pigs. The animals were killed a month after feeding, and though one was negative, the other showed a very heavy infestation. As the two animals were kept in one cage, one of them probably failed to eat any of the salad and thus escaped the disease.

Experiment 2.-Twenty days after experimental infection, blood was drawn from a rabbit and mixed with trichinous pork. Subsequent postmortem examination of the rabbit revealed a heavy infection. The meat mixed with serum was fed to two rats, one of which died nineteen days after feeding and was found to be heavily infested. The second rat died fifty days after feeding and was likewise found to be very heavily infested.

EXPERIMENT 3.-Serum was obtained from a rabbit seven weeks after infection and after it was kept over night in an ice-box was mixed with trichinous meat. Subsequent postmortem examination of the rabbit revealed a heavy infestation. The meat mixed with serum was fed to four rats kept in one cage, and only one of the rats became infected. This one showed a very heavy infestation. The negative resuit with the other rats, as in the case of the guinea-pigs referred to in Experiment 1, was probably due to a failure to eat the meat.

EXPERIMENT 4.-Serum was obtained from a guinea-pig two months after experimental infection, and, after it had been kept over night in an ice-box, was mixed with trichinous meat. Subsequent postmortem examination of the guinea-pig revealed a heavy infestation. The meat mixed with serum was fed to three rats, all of which became infested.

In the foregoing experiments it was found that serum from an animal during the active stage of the disease, or during convalescence when mixed with trichinous meat which is fed to a suitable host, had no effect on the parasites and did not interfere with their developmental cycle. This appears to be directly contrary to Salzer's assertion that trichinous meat mixed with immune serum fails to produce trichinosis.

The direct effect of serum on the larvae was tested a number of times. Trichinous meat was subjected to artificial digestion, and the parasites freed from their cysts were washed in a physiologic sodium chlorid solution, taken up in a capillary pipet and placed in pure serum and in mixtures of serum with various physiologic sodium chlorid solutions. In no case did the serum have any ill effect on the larvae as far as could be ascertained by direct examination. When kept at room temperature, the larvae in the serum succumbed more readily than larvae kept in a 0.7 per cent. sodium chlorid solution, but this was undoubtedly due to the development of bacteria in the serum. The results were similar with "immune" and normal serum. When subjected to a higher temperature ( $37 \mathrm{C}$.) in an incubator, the larvae in the serum and salt solution mixture showed even greater resistance than the larvae in a salt solution without serum, and were still alive and active when the latter were dead. This was doubtless due to the more favorable environment offered by the serum and salt solution mixture.

\section{SUM MARY}

1. Serum from animals convalescent from trichinosis when injected into other animals did not produce immunity to trichinosis in the latter.

2. Trichinous meat mixed with serum from animals during the active or convalescent stage of the disease proved to be still capable of producing the disease.

3. Animals once infected and harboring trichinae in their muscles were not immune to further infection when fed trichinous meat.

4. Serum from a trichinous animal had no observable ill effects on the larvae freed from their cysts by artificial digestion. 
5. None of the results of the experiments appear to be in harmony with the assertions made by Salzer (1916, 1917) concerning the value of serum from convalescent animals as a prophylactic or curative agent in trichinosis.

\section{THE NECESSITY OF A PURE RAW MILK *}

\section{HENRY DWIGHT CHAPIN, M.D. NEW YORK}

Goethe once remarked that blood is a very peculiar juice. We can say the same of milk. Modern physiologic researches have shown that certain glands and secretions of the body have much larger functions than have hitherto been assigned to them. We need only refer to the internal secretions of various glands, formerly unrecognized, that are now known to exert a marvelous influence not only on physical life, but on mental development as well.

These facts have led us to give a closer scrutiny to the more familiar fluids of the body, of which milk is one of the best known, as it constitutes the universal food for the young of all mammalia. From a nutritional standpoint it is recognized as a complete food, containing in itself all the elements required to support life.

While each species of mammalian young is perfectly nourished by the milk of its own mother, the food elements are present in varying proportions in different species, this depending largely on the rapidity of growth of the offspring. But milk is a complicated fluid; with more than one function to perform. This must always be borne in mind when heat or other agents are brought to bear on it. As a general rule, the less milk is manipulated and the closer it is kept to its natural state, the better it will serve the ends for zohich it is intended. Any factor that tends to alter in any way the physical or vital character of milk must be considered as important, and possibly, under certain conditions and in certain ways, may not be of advantage. Some of Nature's foods are evidently intended to be taken in a raw state, as is shown by a certain failure in nutrition which follows when they are subjected to prolonged drying or heating.

There is one familiar but remarkable characteristic of all milks. When collected from the mother they are always in fluid form, but as soon as taken into the stomach of the young, they become more or less solid. This is due to a process of coagulation that takes place in only one of the ingredients-the casein-but which thus always alters the form of the ingested milk. While the carbohydrates and fats in their composition and reaction to the digestive secretions are a good deal alike in different millks, the proteins are essentially different. It is further to be noted that coagulation of the protein of milk takes place in different degrees in the different species.

We are now led to two queries: (1) What is Nature's object in presenting a fluid that always coagulates in the stomach receiving it, and (2) Why do the milks of different species coagulate in different ways? An answer will be found in studying the relation between the milk and the digestive tract that is destined to receive it. While a certain portion of the protein

* Read before the American Association of Medical Milk Commis sions, Brooklyn, June 2, 1917. of all milks coagulates on coming in contact with rennin or rennin and acid, the manner and extent of the coagulation will stand in a direct relation to the proper evolution of the digestive tract of the animal.

Although there are many grades of coagulability in the milks of different animals, we may for practical purposes distinguish three of these grades and consider their significance. The protein may coagulate in a solid, gelatinous or flocculent manner. In the ruminant, herbivorous animals, such as the cow, sheep or goat, the protein coagulates in tough, solid masses that cannot readily escape from the stomach. In these animals, digestion is always largely gastric and the stomach forms 70 per cent. of the digestive tract. Later on, this stomach will be called on largely to digest tough, stringy masses of hay and straw, and the previous exercise on the tough curds of milk develops it for this future work.

In the nonruminant herbivora, such as the mare and the ass, the protein coagulates in gelatinous masses that can easily leave the stomach. There is an object in thus passing the curds quickly along, as in this class of animals digestion is largely intestinal, and the intestine forms about 90 per cent. of the digestive tract. Later on, grasses and grains must be largely digested in the intestinal portion of the tube, and the curd is here also especially adapted to develop a certain part of the intestinal tract for its future work.

In human milk the curd is thrown down in flocculent masses, a form intermediate between the solid and gelatinous types of curd previously noted. While digestion begins in the stomach, it is largely carried on and completed in the intestine, and the stomach forms only about 20 per cent. of the digestive tract. The curd is thus adapted to start the development and motility of the stomach, and finishes by instituting these functions in the bowel, which is destined to play a prominent part in digestion. Here again the curd, as far as form is concerned, furnishes, to a certain extent, an analogue and precursor of the future food of the infant. The curd forms small, flocculent masses, and the food must be separated later into small particles by chewing before digestion can take place to the best advantage.

It thus seems to be a law that the coagulation of the protein of milk always takes place in such a manner as most readily to adapt the digestive tract for its future work, as this function needs special preparation. While a certain amount of protein is present in the milk of all animals and is necessary for tissue building and growth, this protein must not only be coagulable, but must curd in a certain specific way in each species of animal for the proper evolution of their digestive tracts.

To realize what a divergence in the digestive functions has been taking place during the suckling period, imagine an infant, a kitten and a calf all being fed successfully on cow's milk. Here it is evident that at the very beginning of life the difference in their digestive processes is not very great; but wait a year until all three have passed the suckling period. The infant will be just beginning to eat soft food, the kitten will have developed so that it can eat flesh and bones, and the calf will be thriving on grass and hay. In one short year the development and divergence of their digestive tracts has been so great that the natural food of the calf is then wholly unsuited to the kitten or the infant, yet the chemist will find that the food of all three at this time contains the same basic nutritive elements as 